\title{
Using Smartphone Bases Biodevices for Analyzing Physiological, Psychological and Behavioral User's Habits
}

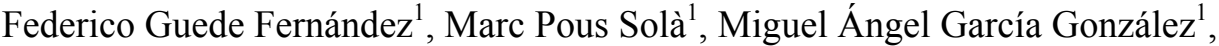 \\ Lluís Capdevila Ortís ${ }^{2}$, Juan Ramos Castro ${ }^{1}$ and Mireya Fernández Chimeno ${ }^{1}$ \\ ${ }^{1}$ Universitat Politècnica de Catalunya, Campus Nord C4 Jordi Girona 1-3, Barcelona, 08034, Spain \\ ${ }^{2}$ Universitat Autònoma de Barcelona, Campus de la UAB Edifici B Bellaterra, Cerdanyola del Vallès, 08193, Spain \\ federico.guede@upc.edu
}

Keywords: Android, Real-time, Biomedical Monitoring, Biosensor.

\begin{abstract}
As a consequence of increasing life expectancy, the promotion of lifestyles that allow aging wellbeing guarantees has acquired great importance in the developed countries. However, the adherence to healthy behaviors in young and adult people remains as a big problem in the community health field. The development of markers of adherence to healthy lifestyles and the evaluation its effectiveness is a goal of many research groups. This paper presents a system for analyzing physiological, psychological and behavioural user's habits using a smartphone and externals biodevices. We use an Android smartphone with an internal tri-axial accelerometer and GPS to monitor physical activity. The smartphone is connected via Bluetooth to a respiratory sensor for breath monitoring. In addition, Android application contains psychological questionnaires to analyze user's mood state and at the same, social interaction is analyzed tracking phone usage and user's social network. Finally, the collected information is sent to a remote server for a long-term processing.
\end{abstract}

\section{INTRODUCTION}

As a consequence of increasing life expectancy, the promotion of lifestyles that allow aging wellbeing guarantees has acquired great importance in the developed countries. However, the adherence to healthy behaviors in young and adult people remains as a big problem in the community health field. The development of markers of adherence to healthy lifestyles and the evaluation its effectiveness is a goal of many research groups.

Healthy lifestyles includes among others, regular exercising and daily physical activity, eating and sleeping habits, and stress control. In order to measure the adherence to these healthy lifestyles, is necessary to have non-intrusive measurement devices, almost transparent for the users, and measurement protocols suitable for every particular application.

The application fields of healthy lifestyle adherence measurements range from monitoring old people habits to the sportsmen and sportswomen quality of training measurement. They also include driver somnolence control, quality of life of workers in shift, or simply individual self-help applications.
Many handheld mobile devices have high speed data transmission capabilities (e.g., 3G, WiFi) and embedded microprocessors with the capability to communicate, using wireless connection (e.g., Bluetooth, NFC), with external sensors. Moreover mobile devices have internal sensors (e.g., accelerometer, gyroscope), supplied by powerful processors and large storage capacities. So, smartphones are strong candidates to be used as biodevices for monitoring parameters related with healthy lifestyle behaviors. From the monitoring point of view, the main advantage of mobile devices, compared to laptops or desktop computers, is that these devices are always handled by the user collecting data during the whole day.

The smartphone market has grown, from being a niche market product segment, becoming a truly mass-market. In 2011, total annual global shipments of smartphones exceeded those of client PCs (including pads) for the first time (Canalys, 2012). In fact, in Europe the smartphone penetration have grown just $47.6 \%$ (comScore, 2012). Regarding operative systems in smartphones, Gartner research shows Android's handset annual sales reached 82,1 million units in first quarter of 2012, in comparison 
with last year sales represents a growth of $123 \%$. This means that Android accounted more than half of all smartphone sales (56.1\%) (Gartner, 2012).

In the area of medicine preventive there some studies about the relation among obesity, nutrition and physical activity (Hensrud, 2010). Another study concludes that sleep loss is associated with having an unhealthy lifestyle.(Ohida et al., 2001).

In several mobile health-care applications, mobile devices are used as gateway for receiving bio-information from medical portable devices and mobile sensors. There, mobile device is responsible of collecting data from sensors, pre-processing the raw signals and transferring the gathered data to remote server (Yang \& Gerla, 2011).

For daily mood assessment, there is an android application that combines accelerometer, sound sensor, light sensor, GPS, and SMS information (Yuanchao et al., 2012). In some applications, mobile device also processes the gathering data and only when mobile detects an emergency, it sends an alert to a server or to social contacts (Sposaro \& Tyson, 2009).

Internal sensors of smartphone are used to monitor heart rate variability using internal accelerometers and recording the seismocardiogram signal (Ramos-Castro et al., 2012).

This work exploits the increasing capabilities of smartphones and its success between the people to promote healthy life habits in people. The purpose of this work is to develop an Android application to analyze physiological, psychological and behavioral personal habits. First we have to collect data to get information about user's life habits. Then application shows some data to user and some information will be sent to a server in order to a third-party can process this information and monitor the level of healthy habits.

\section{MATERIALS AND METHODS}

\subsection{Android OS}

Android is an open-source software stack created for mobile phones and other devices produced by Google. The Android SDK provides the API libraries and developer tools required to build, test, and debug applications. Android applications are written in Java programming language and they run on the Dalvik virtual machine optimized for mobile devices. Android gives an open marketplace for distributing it to Android users called Google Play. Application components are the essential building blocks of an Android application.

There are four different types of application components: Activities, Services, Broadcast receivers and Content providers. Each type serves a distinct purpose and has a distinct lifecycle that defines how the component is created and destroyed. Our application uses activities, for user-interaction, and a broadcast receiver that responds to systemwide broadcast announcements, like Bluetooth device have been found. Activities start on the main application thread, therefore blocking I/O operations or time-consuming processing have to be executed in our own threads to avoid blocking the activity.

The layout is the architecture to design the user interface (UI) in an activity. Layouts may be declared using XML files or using Java code in runtime. The main advantage of using XML files to design the UI is that enables to separate the presentation tier of the Activity logic tier that controls its behavior (Google Inc., 2012a). This improves the maintenance for later works. Therefore, our application uses XML files.

We have selected Android OS to develop our application for the following reasons: Android is an open-source software and development tools are free of charge; it also allows to interact easily with internal sensors such GPS to track physical activity, accelerometer to activity monitoring; it provides simplified and well-documented Bluetooth, WiFi APIs to communicate with external sensors. Moreover there are several APIs to develop quickly powerful applications.

\subsection{Internal Sensor}

Nowadays, the smartphones have integrated many internal sensors, such as accelerometer, magnetic field sensor, light sensor, GPS and proximity sensor. The main advantage of these sensors is that they don't require an external communication channel, such as Bluetooth of WiFi improves the battery consumption and the facility to access the data provided by the sensor.

For example, the accelerometer has been employed in several applications to detect human movement detection, to characterize physical activity (Shin et al., 2010) recognizing if the user is walking, going upstairs or running (Brezmes et al., 2009). In our application we have developed filters to reduce noise; Butterworth second order high-pass filter is implemented. The accelerometer gives three axis forces applied on the smartphone, in the implemented application the contribution of earth gravity have been removed. 
Another sensor available in most of the mobile phone is the light sensor. This can be used to detect user light exposure. Nevertheless this sensor is not useful to measure the level of sun exposure because if the mobile phone is in the pocket or bag, do not provide information of user exposition. For this reason this sensor isn't useful for this propose.

So, in function of the application, several external sensors must be connected to the device, but this is not a problem with the level of connectivity of these devices

\subsection{Breathing Monitoring}

Breathing is a good indicator of somnolence (Rodríguez-Ibañez et al., 2011). In order to record the respiration signal we use an external monitoring system (Bioplux Respiband sensor). It consists in a respiratory sensor based on an inductive band to measure thoracic effort. This sensor has the embedded electronic to calculate breath signal. Moreover, it has a Bluetooth communication module that makes possible to connect it to smartphone. Then, mobile application may monitor the breath signal in real time. Mobile device parses data received and displays breath signal to user. Bluetooth communication uses Serial Port Profile (SPP) that defines how to set up virtual serial ports and connect two Bluetooth enabled devices. Once Respiband has been connected to smartphone, it starts to send data continuously to a frequency configured between $36 \mathrm{~Hz}$ and $1000 \mathrm{~Hz}$. In our application the sampled frequency selected is $40 \mathrm{~Hz}$. This frequency is enough to characterize properly the breath rate signal and reduce power consumption due to Bluetooth transmission.

Android application has an activity to manage Bluetooth connection. This activity lists paired devices and allows to scan new devices, to pair and to connect. Once paired an own thread is started to establish RFCOMM channel and to maintain the communication. This thread is continuously reading new incoming data in real-time. When an incoming packet is detected, the application parses it to extract one byte of CRC and three bytes of value data. Firstly CRC is checked to verify data integrity and secondly, the application sends measured breath value to UI to plot it. The data is visualized using an open source charting library called AChartEngine (4ViewSoft, 2012). If the Bluetooth connection is lost, the application detects it and tries to reconnect continuously since user exit

\subsection{User Location Monitoring}

Android application uses location provider to track exercise, using speed data, distance traveled and the altimeter.

Android OS provide different methods to determine the device's current location, they are called location providers, and each has its different characteristics. Each location provider offers different capabilities, including differences in power consumption, accuracy, and the ability to determine altitude or speed or heading information. The three different locations providers are GPS_Provider, Network Provider, Passive Provider. Therefore each one is suitable to work over different conditions. Using the Android Location Manager API we can find available location providers, obtain device's current location, track movement, set proximity alerts for detecting movement into and out of a specified area.

GPS_provider determines location using satellites. Depending on conditions, this provider may take a while to return a location fix, this time is called Time to First Fix (TTFF.) This time can be cut down substantially by employing Assisted GPS (A-GPS). A-GPS uses the mobile network to transmit the precise GPS satellite orbit and clock information to a mobile device. Then the device will know the approximate location of the GPS satellites in its line of sight. Reducing the number of frequency bins which must be searched to acquire the signal reduces the TTFF (Jarvinen et al., 2002).

The main limitation of GPS provider is that it only can work in outdoor environment, because in indoors GPS signals don't reach. Moreover this provider consumes more battery than the others. Furthermore, GPS provider provides the most accurate location data and it is unique that has ability to determine altitude information. Therefore, we need use GPS_provider to track people exercise properly.

Our Android application has a dedicated activity that employs GPS_provider to obtain the user's location. Using information of location obtained, in real-time, our application calculates main parameters concerning a healthy behavior such as total traveled distance, average speed and time spent. Apart from displaying the parameters explained before the application shows the route followed by the user using maps. There are several libraries that provide maps, we used Google Maps API (Google Inc., $2012 \mathrm{~b}$ ) and an open alternative called OSMDroid (Gramlich, 2012) to display OpenStreetMap (OSM). The usage of both APIs is quite similar for 
programmer. Android application uses overlays to draw followed route with points and lines. The main disadvantage of Google Maps that it has a prize policy of use otherwise OSM is totally free of use.

We have considered that more times while user is doing exercise he is looking away from the screen. Then for a better human-interaction Android provides text-to-speech, application speaks to user to notify each certain time period distance traveled and time spent. Text to speech API provided for Android it is used to implement this.

\subsection{Social Interaction Tracker}

Social interaction is important to human health. In fact, individuals with adequate social relationships have a $50 \%$ greater likelihood of survival compared to those with poor or insufficient social relationships. The influence of quality and quantity of individual's social relationships on the risk of death are comparable with well-established risk factors for mortality such as smoking and alcohol consumption and they exceed other many risk factors such as physical inactivity and obesity. (Holt-Lunstad et al., 2010).

On the other hand, one of the most important concerns associated with mobile phone use is that it may become uncontrolled or excessive, which has an impact upon daily living. Sleep disturbance is among the most common negative outcomes resulting from overuse of the mobile phone (Thomée et al., 2011). Furthermore, smartphones allows people to engage in a wide range of online activities - such as Internet surfing, managing email, playing video games or involvement in social networks (e.g., Facebook, Twitter). Therefore, mobile phone addiction should be conceptualized within a broader spectrum of "cyber addictions" that covers a wide range of behaviors relying on online activities and/or activities involving communication among individuals through technological devices. Social network addictions is one of the most frequent "cyber additions" (Billieux, 2012).

Android application can collect some information about social interaction. On the one hand, in order to track incoming and outgoing phone calls and SMS, the application registers BroadcastReceiver to track the phone usage. This information is useful to evaluate social relationship. Nowadays new social networks based on Internet are very used to social interaction; Facebook and Twitter are currently the most popular ones. Our application uses Facebook (Facebook, 2012) and Twitter API (Yamamoto, 2011) for Android parsing
JSON data received to collect information about user's social network like number of friends, frequency update status, messages.

\subsection{Psychological State Evaluation}

Professionals and researchers are working in the relationships existing between physical activity and mood state. The most popular instrument for mood state evaluation is POMS (McNair, 1984).

Questionnaire is a subjective method to evaluate mood sate. Android application implements a test of POMS with fifteen questions to evaluate user's state of mood obtaining the components of stress, depression, hostility, vigor, and fatigue (Andrade et al., 2010). The user has to indicate how the user feels in $0-10$ scale where 0 means not all and 10 very. There are three questions for mentioned component to avoid random answers. In the beginning and at the end of respiratory acquisition the application launches the questionnaire to be filled by the user. The results of the test answered by the user provide useful information to study the relationship between psychological, physiological and behavioral habits.

\section{RESULTS}

Android application will forward physiological, psychological and behavioral data obtained to a remote server for a long term processing via Android TCP socket when application finishes. It uses Wi-Fi connection to reduce connection cost and to increase rate transmission.

The breath signal acquired using Respiband external sensor is monitored in real-time by the application developed. In Figure 1, a screen captured shows a XY graph displaying in $y$-axis the value of amplitude and $\mathrm{X}$-axis the samples. This graph is refreshed when a packet of data is received. Usually there is a packet for each sample of Respiband; its sample rate is $40 \mathrm{~Hz}$. The designed layout included a graph to plot respiratory signal on time domain in real-time. To plot the data the open source charting library called AChartEngine is used.

For user's location monitoring, application shows the route tracked displaying it over a map. In our application we have used two technologies that provide maps: GoogleMaps API (Figure 2a) and OpenStreetMap API (Figure 2b). In addition, the application calculates and displays information about distance traveled, average speed and time spent. In order to improve the accuracy of this 
information, user can pause and resume the tracking, this is essential to avoid wrong results.

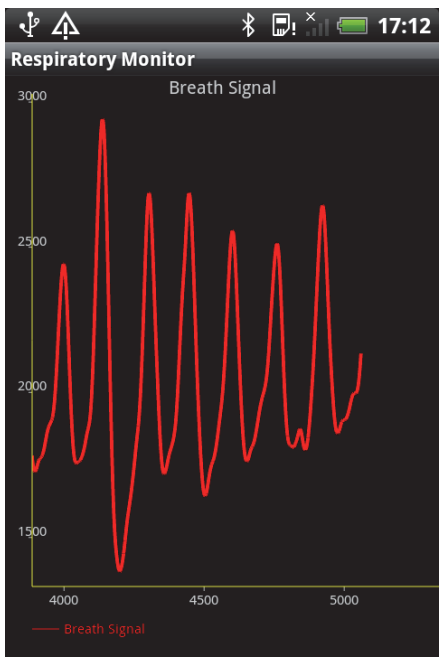

Figure 1: Mobile application screenshot: graphical breath signal represented in real-time.

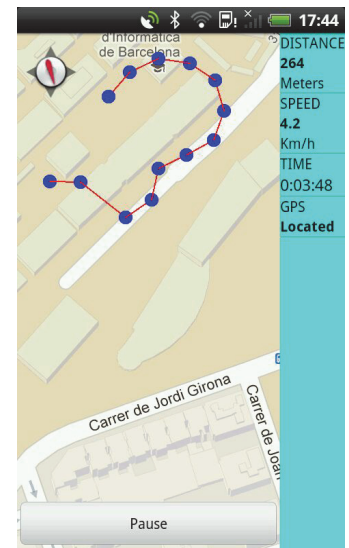

(a)

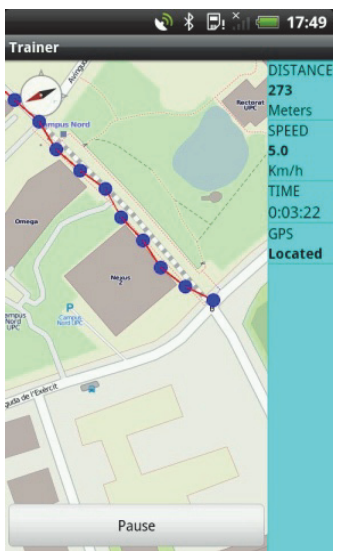

(b)
Figure 2: Screenshots: route tracked using Google Maps (a). Route tracked using OpenStreetMap (b).

The application has provided us useful types of data to analyze social activity. In the application the number and time when an SMS is sent or received has been monitored. It also has allowed tracking the duration, the number and the time of outgoing and incoming phone calls. Concerning the social networks, the application has been capable to analyze the activity on Facebook and Twitter. The application has obtained the number of friends that is associated with the contact network size. In addition to measure the social activity, the wall panel, messages and notifications have been checked. On Twitter social network published tweets by the user, mentions, direct messages, the number of followers and "following" have been quantified.
To evaluate the mood state in the application a test with fifteen questions is launched in the beginning and at the end (Figure 3a). The five components of the mood state mentioned in section 2.6 have calculated each time when the user completes the questionnaire (Figure $3 b$ ).

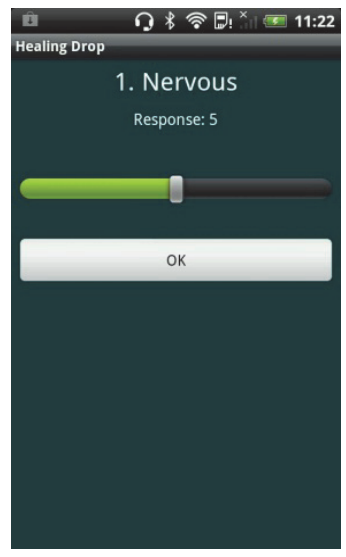

(a)

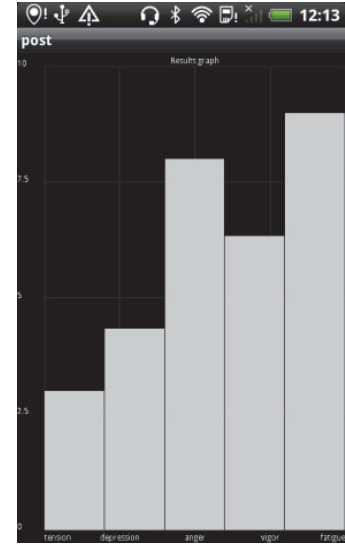

(b)
Figure 3: Screenshots: a question of the test to evaluate the mood state (a). Graph that represents the five components resulted of a completed test (b).

The results obtained are stored in a database proving us information of the psychological state when the user is monitored.

\section{CONCLUSIONS}

A smartphone based on biodevice for healthy lifestyle adherence evaluation has been developed. The Android application developed collects the information about different variables (activity, respiration, social activity, etc.) and processes them to measure the adherence to healthy lifestyles. The data are sent to a remote server for further processing to get results to improve user lifestyle.

\section{ACKNOWLEDGEMENTS}

This work has been partially funded by the Spanish MINISTERIO DE CIENCIA E INNOVACIÓN. Proyecto IPT-2011-0833-900000. Healthy Life style and Drowsiness Prevention-HEALING DROP.

\section{REFERENCES}

4ViewSoft. (2012). AChartEngine: Charting Library for 
Android (Version 1.0.0) [Software]. Available from http://code.google.com/p/achartengine/downloads/list

Andrade, E., Arce, C., Torrado, J., Garrido, J., De Francisco, C., and Arce, I., (2010). Factor structure and invariance of the POMS mood state questionnaire in spanish. Spanish Journal of Psychology, 13(1), 444452.

Billieux, J., (2012). Problematic use of the mobile phone: A literature review and a pathways model. Current Psychiatry Reviews, 8(4), 299-307. doi: 10.2174/157340012803520522.

Brezmes, T., Gorricho, J. L. and Cotrina J., (2009). Activity recognition from accelerometer data on a mobile phone. In Proceedings of the IWANN '09, 796799.

Canalys, (2012). Smart phones overtake client PCs in 2011. Retrieved February 3, 2012, from http://www.canalys.com/newsroom/smart-phonesovertake-client-pcs-2011.

comScore, (2012). Number of european smartphone users accessing news surges 74 percent over past year. Retrieved March 22, 2012, from http://www.comscore.com/Insights/Press_Releases/20 12/3/Number_of_European_Smartphone_Users_Acces sing_News_Surges_74_Percent_Over_Past_Year.

Facebook, (2012). Android Tutorial. Retreive $\bar{d}$ May 18, 2012, from https://developers.facebook.com/ docs/mobile/android/build/

Gartner. (2012). Gartner says worldwide sales of mobile phones declined 2 percent in first quarter of 2012; previous year-over-year decline occurred in second quarter of 2009. Retrieved May 16, 2012, from http://www.gartner.com/it/page.jsp?id=2017015.

Google Inc. (2012a). Android developer guide: Layouts. Retrieved May 11, 2012, from http://developer.android.com/guide/topics/ui/declaring -layout.html.

Google Inc. (2012b). Google Maps Android API External Library. Retreived June 1, 2012, from https://developers.google.com/maps/documentation/an droid/index

Gramlich, N. (2012). OSMDroid: OpenStreetMap-Tools for Android (Version 3.0.8) [Software]. Avaliable from http://code.google.com/p/osmdroid/

Holt-Lunstad, J., Smith, T. B., \& Layton, J. B., (2010). Social relationships and mortality risk: A metaanalytic review. PLoS Medicine, 7(7) doi: 10.1371/journal.pmed.1000316

Jarvinen, J., DeSalas, J. \& LaMance, J., (2002). Assisted GPS: A Low-Infrastructure Approach. Retrieved March 1, 2002, from http://www.gpsworld.com/wpcontent/uploads/2012/09/gpsworld_Innovation_0302.p df

McNair, D., (1984). Citation classic - manual for the profile of mood states. Current Contents/social \& Behavioral Sciences, (27), 20-20.

Ohida, T., Kamal, A. M. M., Uchiyama, M., Kim, K., Takemura, S., Sone, T and Ishii, T., (2001) The influence of lifestyle and health status factors on sleep loss among the Japanese general population. SLEEP, 24(3), 333-338.

Ramos-Castro, J., Moreno, J., Miranda-Vidal, H., GarcíaGonzález, M .A., Fernández-Chimeno, M., Rodas, G. \& Capdevila, Ll., (2012). Heart Rate Variability analysis using a Seismocardiogram signal. In Engineering in Medicine and Biology Society, EMBC 2012. Accepted.

Rodriguez-Ibanez, N., Garcia-Gonzalez, M. A., Fernandez-Chimeno, M., \& Ramos-Castro, J., (2011). Drowsiness detection by thoracic effort signal analysis in real driving environments. 33rd Annual International Conference of the IEEE Engineering in Medicine and Biology Society, EMBS 2011, Boston, MA. 6055-6058. doi: 10.1109/IEMBS.2011.6091496

Shin, J., Shin, D., Shin, D., Her, S., Kim, S., \& Lee, M., (2010). Human movement detection algorithm using 3axis accelerometer sensor based on low-power management scheme for mobile health care system (Hualien ed.) doi: 10.1007/978-3-642-13067-0_12

Sposaro, F., \& Tyson, G., (2009). iFall: An android application for fall monitoring and response. 31st Annual International Conference of the IEEE Engineering in Medicine and Biology Society: Engineering the Future of Biomedicine, EMBC 2009, Minneapolis, MN. 6119-6122. doi: 10.1109/IEMBS.2009.5334912

Thomée, S., Härenstam, A. \& Hagberg, M., (2011). Mobile phone use and stress, sleep disturbances, and symptoms of depression among young adults - A prospective cohort study. BMC Public Health, 11 doi: 10.1186/1471-2458-11-66

Yamamoto, Y., (2011). Twitter4j: an open-sourced Java library for the Twitter API. (Version 2.0.4) Avaliable from http://twitter4j.org/en/index.html

Yang, S. \& Gerla, M., (2011). Personal gateway in mobile health monitoring. 2011 9th IEEE International Conference on Pervasive Computing and Communications Workshops, PERCOM Workshops 2011, Seattle, WA. 636-641. doi: 10.1109/PERCOMW.2011.5766966

Yuanchao, M., Bin, X. Yin, B., Guodong, S. and Run, Zhu, (2012). Daily Mood Assessment Based on Mobile Phone Sensing. In Wearable and Implantable Body Sensor Networks, 2012 Ninth International Conference on, 142-147. 